\title{
Recovery of Schell-model partially coherent beams
}

\author{
José A. Rodrigo* and Tatiana Alieva \\ Universidad Complutense de Madrid, Facultad de Ciencias Físicas, Ciudad Universitaria s/n, Madrid 28040, Spain \\ ${ }^{*}$ Corresponding author: jarmar@fis.ucm.es
}

Received December 13, 2013; revised January 14, 2014; accepted January 14, 2014; posted January 15, 2014 (Doc. ID 203102); published February 12, 2014

\begin{abstract}
Partially coherent light is often preferable to its completely coherent counterpart in applications such as imaging, sensing, and free-space optical communications. To fully exploit its advantages, techniques able to retrieve information carried by the beam are required. Here, we develop and experimentally demonstrate a phase-space optics technique for complete spatial analysis of widely used Schell-model beams. It allows for fast information recovery and can be applied for quantitative phase imaging of objects under partially coherent illumination. (C) 2014 Optical Society of America

OCIS codes: (030.0030) Coherence and statistical optics; (070.2575) Fractional Fourier transforms; (100.3010) Image reconstruction techniques; (100.5070) Phase retrieval; (090.1995) Digital holography.

http://dx.doi.org/10.1364/OL.39.001030
\end{abstract}

The further development of imaging techniques requires a more realistic model of illumination as partially coherent light instead of its limiting cases: the coherent or incoherent light used so far. Moreover, partially coherent beams have important advantages with respect to completely coherent ones in other relevant applications such as lithography, plasma confinement, and free-space communication, to name a few. The description of partially coherent light is inherently complex, and its characterization is difficult even in the quasi-monochromatic scalar paraxial approximation. Indeed, a two-dimensional (2D) partially coherent beam is described by a complexvalued $4 \mathrm{D}$ function, that is, mutual intensity (MI) defined as $\Gamma\left(\mathbf{r}_{1}, \mathbf{r}_{2}\right)=\left\langle f\left(\mathbf{r}_{1}\right) f^{*}\left(\mathbf{r}_{2}\right)\right\rangle$, where: $\mathbf{r}_{1,2}$ is the position vector in a plane transverse to the beam propagation direction and $\langle\cdot\rangle$ stands for ensemble averaging. The coherent case corresponds to $\Gamma_{c}\left(\mathbf{r}_{1}, \mathbf{r}_{2}\right)=f\left(\mathbf{r}_{1}\right) f^{*}\left(\mathbf{r}_{2}\right)$. Schell-model partially coherent beams (SMBs) [1], described by $\Gamma\left(\mathbf{r}_{1}, \mathbf{r}_{2}\right)=f\left(\mathbf{r}_{1}\right) f^{*}\left(\mathbf{r}_{2}\right) \gamma\left(\mathbf{r}_{1}-\mathbf{r}_{2}\right)=\Gamma_{c}\left(\mathbf{r}_{1}, \mathbf{r}_{2}\right)$ $\gamma\left(\mathbf{r}_{1}-\mathbf{r}_{2}\right)$, where $\gamma(\mathbf{r})$ is an equal-time complex degree of spatial coherence (DoC), are often used in practical applications. This kind of beam is generated, for example, when a partially coherent plane wave characterized by $\gamma(\mathbf{r})$ propagates through an object described by a complex modulation function, $f(\mathbf{r})$, as sketched in Fig. 1(a). We recall that, according with the van Cittert Zernike theorem, a partially coherent plane wave can be created by collimating light emitted from an incoherent source with intensity distribution $I_{\text {inc }}(\mathbf{r})$. Specifically, $\gamma(\mathbf{r}) \propto \int I_{\text {inc }}\left(\mathbf{r}^{\prime}\right) \exp \left(-i 2 \pi \mathbf{r}^{\prime} \mathbf{r} / \lambda \mathbf{f}_{\mathrm{cl}}\right) \mathrm{d} \mathbf{r}^{\prime}$, where $\lambda$ is the wavelength and $f_{\mathrm{cl}}$ is the focal length of the collimating lens [2]. Moreover, the generalization of structurally stable and spiral coherent beams to the partially coherent case is also described by the Schell model $[3,4]$. In the last decades, special attention has been paid to coherent vortex beams that carry orbital angular momentum (OAM) useful for different applications such as optical tweezers and free-space communications [5]. On the other hand, the SMB vortex has been found more robust than its coherent counterpart to distortions caused by turbulent atmosphere [,ㅜㄱ. We underline that the valuable information carrie by the SMB is often encoded into $f(\mathbf{r})$, while $\gamma(\mathbf{r})$ in most, but not all, cases is known.
In this Letter we propose and experimentally demonstrate an iterative MI retrieval technique for arbitrary SMBs with a priori known or unknown DoC. It is based upon the measurement of the intensity distributions of the beam transformed by a fractional Fourier transform (FrFT) system, which has been demonstrated to be powerful for beam characterization [요, $\underline{9}$. As an example, we analyze partially coherent vortex beams by reconstructing both their $f(\mathbf{r})$ and DoC.

We recall that the FrFT of a complex field amplitude $f\left(\mathbf{r}_{i}\right)$ for transformation angles $\left(\alpha_{x}, \alpha_{y}\right)$ is expressed as

$$
\mathcal{F}^{\alpha_{x}, \alpha_{y}}\left[f\left(\mathbf{r}_{i}\right)\right]\left(\mathbf{r}_{o}\right)=\int f\left(\mathbf{r}_{i}\right) K^{\alpha_{x}, \alpha_{y}}\left(\mathbf{r}_{i}, \mathbf{r}_{o}\right) \mathrm{d} \mathbf{r}_{i},
$$

which kernel is separable, $K^{\alpha_{x}, \alpha_{y}}\left(\mathbf{r}_{i}, \mathbf{r}_{o}\right)=K^{\alpha_{x}}\left(x_{i}, x_{o}\right) K^{\alpha_{y}}$ $\left(y_{i}, y_{o}\right)$, with $K^{\alpha_{q}}\left(q_{i}, q_{o}\right)=\sqrt{\sigma / i \sin \alpha_{q}} \exp \left(i \pi \sigma\left(q_{o}^{2}+q_{i}^{2}\right)\right.$ $\cot \alpha_{q}-i 2 \pi \sigma q_{i} q_{o} \csc \alpha_{q}$ ), with $q$ being a placeholder and $\alpha_{q} \in[0,2 \pi]$. Here $\sigma$ is a normalization factor, and $\mathbf{r}_{i, o}=\left[x_{i, o}, y_{i, o}\right]^{t}$ is given at input $(i)$ and output $(o)$ planes of the FrFT system. Note that $\mathcal{F}^{(\pi / 2),(\pi / 2)}$ corresponds to the Fourier transform, while by applying $\mathcal{F}^{0,0}$ the $f\left(\mathbf{r}_{i}\right)$ is obtained. In the case of the partially coherent light, with $\Gamma\left(\mathbf{r}_{1}, \mathbf{r}_{2}\right)$ at the input plane of the FrFT system, the intensity distribution at its output plane is expressed as it follows:

$$
\begin{aligned}
I_{\mathrm{pc}}^{\alpha_{x}, \alpha_{y}}\left(\mathbf{r}_{o}\right)= & \iint \Gamma\left(\mathbf{r}_{1}, \mathbf{r}_{2}\right) K^{\alpha_{x}, \alpha_{y}}\left(\mathbf{r}_{1}, \mathbf{r}_{o}\right) \\
& \times K^{-\alpha_{x},-\alpha_{y}}\left(\mathbf{r}_{2}, \mathbf{r}_{o}\right) \mathrm{d} \mathbf{r}_{1} \mathrm{~d} \mathbf{r}_{2},
\end{aligned}
$$

while in the coherent case it is written as $I_{c}^{\alpha_{x}, \alpha_{y}}\left(\mathbf{r}_{o}\right)=\left|\mathcal{F}^{\alpha_{x}, \alpha_{y}}\left[f\left(\mathbf{r}_{i}\right)\right]\left(\mathbf{r}_{o}\right)\right|^{2}$. A video rate measurement of these intensity distributions can be performed using the programmable FrFT setup reported in [10], which is sketched in Fig. 1(b). This setup comprises a CCD camera and two variable lenses, addressed into spatial light modulators (SLMs, Holoeye LCR-2500, 8-bit gray-level, pixel size of $19 \mu \mathrm{m}$ ), whose focal lengths are given as a function of the angles $\left(\alpha_{x}, \alpha_{y}\right)$ in a $\pi$ interval. In this setup $\sigma=1 / 2 \lambda z$, where $\lambda=532 \mathrm{~nm}$ is the wavelength and $z=50 \mathrm{~cm}$ is the distance between the lenses.

In general, the MI of an arbitrary partially coherent beam can be recovered from the set of intensity distributions $\left\{I_{\mathrm{pc}}^{\alpha_{x}, \alpha_{y}}\left(\mathbf{r}_{o}\right)\right\}$. However, in the case of a SMB not all 
(a)

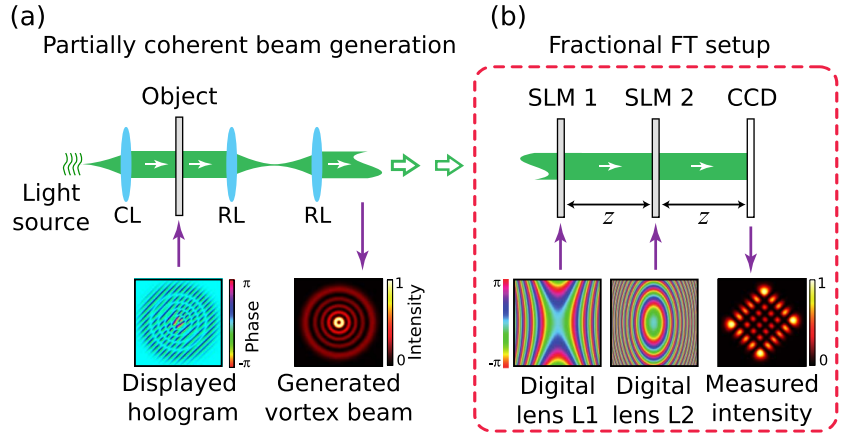

Fig. 1. (a) Setup for generation of SMBs: light from an incoherent source is collimated by a lens (CL) to illuminate the object (hologram). The studied SMB is projected by the relay lenses (RL) into the FrFT setup sketched in (b).

the elements of this set are needed because such a beam has fewer degrees of freedom than a general one. Specifically, here we consider the reduced set $\left\{I_{\mathrm{pc}}^{\alpha,-\alpha}\left(\mathbf{r}_{o}\right)\right\}$ corresponding to the antisymmetric FrFT (aFrFT), $\mathcal{F}^{\alpha,-\alpha}$, further denoted as aFrFT, where the angle $\alpha$ belongs to a $\pi / 2$ interval. This set will be shortly referred to as $\left\{I_{\mathrm{pc}}^{\alpha}\left(\mathbf{r}_{o}\right)\right\}$. An astigmatic transformation such as the aFrFT increases reconstruction accuracy and robustness against noise as well as speeds up the convergence of iterative phase retrieval algorithms [11]. In contrast to conventional iterative algorithms the ones based on astigmatism allows for a unique solution for vortex beam recovery [11, $\underline{12}]$.

Introducing new variables, $\mathbf{r}=\mathbf{r}_{1}-\mathbf{r}_{2}$ and $\mathbf{R}=$ $\left(\mathbf{r}_{1}+\mathbf{r}_{2}\right) / 2$, Eq. (2) for the case of aFrFT is expressed as

$$
\begin{aligned}
I_{\mathrm{pc}}^{\alpha}\left(\mathbf{r}_{o}\right)= & \left(\frac{\sigma}{\sin \alpha}\right)^{2} \iint f\left(\mathbf{R}+\frac{\mathbf{r}}{2}\right) f^{*}\left(\mathbf{R}-\frac{\mathbf{r}}{2}\right) \\
& \times \exp [i 2 \pi \sigma \cot \alpha(X x-Y y)] \mathrm{d} \mathbf{R} \\
& \times \gamma(\mathbf{r}) \exp \left[i 2 \pi \sigma \csc \alpha\left(-x_{o} x+y_{o} y\right)\right] \mathrm{d} \mathbf{r} .
\end{aligned}
$$

Since for the coherent case the intensity $I_{c}^{\alpha}\left(\mathbf{r}_{o}\right)=$ $\left|\mathcal{F}^{\alpha,-\alpha}[f]\left(\mathbf{r}_{o}\right)\right|^{2}$ is also obtained from the latter expression with $\gamma(\mathbf{r})=1$, one can rewrite Eq. (3) as a convolution between the coherent intensity $I_{c}^{\alpha}\left(\mathbf{r}_{o}\right)$ and $\tilde{\gamma}_{\alpha}\left(\mathbf{r}_{o}\right)$ :

$$
I_{\mathrm{pc}}^{\alpha}\left(\mathbf{r}_{o}\right)=I_{c}^{\alpha}\left(\mathbf{r}_{o}\right) \otimes \tilde{\gamma}_{\alpha}\left(\mathbf{r}_{o}\right)=\int I_{c}^{\alpha}\left(\mathbf{r}_{o}^{\prime}\right) \tilde{\gamma}_{\alpha}\left(\mathbf{r}_{o}-\mathbf{r}_{o}^{\prime}\right) \mathrm{d} \mathbf{r}_{o}^{\prime},
$$

where $\tilde{\gamma}_{\alpha}\left(\mathbf{r}_{o}\right)$ is the scaled antisymmetric FT, $\mathcal{F}^{-(\pi / 2),(\pi / 2)}$, of the input DoC: $\tilde{\gamma}_{\alpha}\left(\mathbf{r}_{o}\right)=\mathcal{F}^{-(\pi / 2),(\pi / 2)}[\gamma(\mathbf{r})]\left(\mathbf{r}_{o} \csc \alpha\right)$. Similar relations between the intensity distribution of coherent and partially coherent light in the FT and Fresnel domains have been studied in $[2,13]$.

Let us first consider the recovery of the wavefield, $f(\mathbf{r})$, when the DoC, $\gamma(\mathbf{r})$, is known. In this case the iterative aFrFT algorithm comprises two loops labeled with index $m=1, \ldots M$ and $n=1, \ldots, N$, where $M$ is the number of measured constraint intensities denoted as $I_{\mathrm{pc}}^{\alpha=\alpha(m)}\left(\mathbf{r}_{o}\right)$, and $M \times N$ is the total number of iterations. The wavefield at the input plane is $g_{n}\left(\mathbf{r}_{i}\right)$, whereas at the detector plane (e.g., CCD camera) it is $W_{m, n}\left(\mathbf{r}_{o}\right)=$ $\mathcal{F}^{\alpha(m),-\alpha(m)}\left[g_{n}\left(\mathbf{r}_{i}\right)\right]\left(\mathbf{r}_{o}\right)$. Without loss of generality, an arbitrary Gaussian function can be used as the initial estimate, $g_{1}\left(\mathbf{r}_{i}\right)$. The whole process is described as follows: (i) A new estimate of $W_{m, n}\left(\mathbf{r}_{o}\right)$ is obtained from the current version of $g_{n}\left(\mathbf{r}_{i}\right)$ and then is replaced by the updatedversion,

$$
W_{m, n}^{\prime}\left(\mathbf{r}_{o}\right)=W_{m, n}\left(\mathbf{r}_{o}\right) \sqrt{I_{\mathrm{pc}}^{\alpha(m)}}\left(\mathbf{r}_{o}\right) / \sqrt{\mathcal{I}_{\mathrm{pc}, n}^{\alpha(m)}}\left(\mathbf{r}_{o}\right)
$$

where the current estimate of the intensity is $\mathcal{I}_{\mathrm{pc}, n}^{\alpha(m)}\left(\mathbf{r}_{o}\right)=$ $\mathcal{I}_{c, n}^{\alpha(m)}\left(\mathbf{r}_{o}\right) \otimes \tilde{\gamma}_{\alpha(m)}\left(\mathbf{r}_{o}\right)$, being $\quad \mathcal{I}_{c, n}^{\alpha(m)}\left(\mathbf{r}_{o}\right)=\left|W_{m, n}\left(\mathbf{r}_{o}\right)\right|^{2}$. (ii) Then, using the additive properties of the FrFT, the next estimate of the field for the angle $\alpha(m+1)$ is calculated as $W_{m+1, n}\left(\mathbf{r}_{o}\right)=\mathcal{F}^{\Delta \alpha(m),-\Delta \alpha(m)}\left[W_{m, n}^{\prime}\left(\mathbf{r}_{i}\right)\right]\left(\mathbf{r}_{o}\right)$, where $\Delta \alpha(m)=$ $\alpha(m+1)-\alpha(m)$. Applying Eq. (5), the field $W_{m+1, n}\left(\mathbf{r}_{o}\right)$ is transformed into $W_{m+1, n}^{\prime}\left(\mathbf{r}_{o}\right)$. (iii) The procedure described in (ii) is performed using the rest of the measured intensities until $m=M$. Then $W_{M, n}^{\prime}\left(\mathbf{r}_{o}\right)$ is inverted to obtain an updated estimate of the wavefield: $g_{n+1}\left(\mathbf{r}_{i}\right)=$ $\mathcal{F}^{-\alpha(M), \alpha(M)}\left[W_{M, n}^{\prime}\left(\mathbf{r}_{o}\right)\right]\left(\mathbf{r}_{i}\right)$; The process (i)-(iii) is iterated over the index $n$ and stops (at $n=N$ ) when the estimated intensities $\mathcal{I}_{\mathrm{pc}, N}^{\alpha(m)}\left(\mathbf{r}_{o}\right)$ coincide with the measured ones or when algorithm convergence is reached. Note that in the completely coherent case, $\tilde{\gamma}_{\alpha}\left(\mathbf{r}_{o}\right) \rightarrow \delta\left(\mathbf{r}_{o}-\mathbf{r}_{o}^{\prime}\right)$, expression Eq. (5) reducesto the usualmodulus constraint that replaces the amplitude by the measured one while retaining the phase.

To estimate the accuracy of the reconstructed signal $g(\mathbf{r})$, the normalized inner product between it and the reference signal $f(\mathbf{r})$ can be used: $\eta=\mid \int f^{*}(\mathbf{r})$ $\left.g(\mathbf{r}) \mathrm{d} \mathbf{r}\right|^{2} / \int|g(\mathbf{r})|^{2} \mathrm{~d} \mathbf{r} \int|f(\mathbf{r})|^{2} \mathrm{dr} ;$ see [14]. Note that this correlation parameter equals 1 when $g(\mathbf{r})=f(\mathbf{r})$. It is well suited for comparing two complex-valued signals because it takes into account their intensity and phase. In the case of unknown reference $f(\mathbf{r})$, the rootmean-square (RMS) error between reconstructed and measured intensity distributions can be considered to evaluate the accuracy of the retrieved signal $g(\mathbf{r})$. Here, we considered the normalized RMS error $(\varepsilon)$ given by $\varepsilon^{2}=\sum_{q=1}^{Q}\left[\sqrt{c_{q}^{\prime}}-\sqrt{c_{q}}\right]^{2} / \sum_{q=1}^{Q} c_{q}$, where $c_{q}^{\prime}=\mathcal{I}_{p c, n}^{\alpha(m)}(q)$, $c_{q}=I_{p c}^{\alpha(m)}(q)$, with $q$ being the pixel index.

To test the technique, we consider coherent and partially coherent vortex beams corresponding to a helical Laguerre-Gaussian mode: $\operatorname{LG}_{p, l}(\mathbf{r}, w)=L_{p}^{|l|}\left(2 \pi r^{2} / w^{2}\right)$ $\exp \left(-\pi r^{2} / w^{2}\right) \exp (i l \theta)$, where $r^{2}=x^{2}+y^{2}, \tan \theta=y$ / $x, w$ is the beam waist, and $L_{p}^{|l|}$ is the Laguerre polynomial with radial index $p$ and azimuthal index $l$ (i.e., topological charge, with $l \hbar$ being the OAM per photon). Specifically, the signal $f(\mathbf{r})=\mathrm{LG}_{4,1}(\mathbf{r}, w=0.73 \mathrm{~mm})$ was encoded into a computer-generated hologram displayed by a SLM (Holoeye PLUTO, 8-bit gray-level, pixel size of $8 \mu \mathrm{m})$; see Fig. 1(a). The vortex beams were generated by illuminating the hologram with a collimated coherent and partially coherent laser beam [9], correspondingly. In our case, the partially coherent illumination was obtained by collimation of the laser light scattered by a rotating ground glass diffuser. This illumination beam has a DoC $\gamma(\mathbf{r})=\exp \left(-\pi \mathbf{r}^{2} / 2 w_{c}^{2}\right)$, where $w_{c}=0.45 \mathrm{~mm}$ [9]. Therefore the resulting SMB is described by $\Gamma\left(\mathbf{r}_{1}, \mathbf{r}_{2}\right)=$ $\mathrm{LG}_{4,1}\left(\mathbf{r}_{1}, w\right) \mathrm{LG}_{4,1}^{*}\left(\mathbf{r}_{2}, w\right) \exp \left(-\pi\left(\mathbf{r}_{1}-\mathbf{r}_{2}\right)^{2} / 2 w_{c}^{2}\right)$. The CCD camera (8-bit gray-level, pixel size of $4.6 \mu \mathrm{m}$ ) acquired all the constraint images $(Q=1024 \times 1024$ pixels $)$ at video rate; see Media 1.

The reconstruction of the coherent vortex $L_{4,1}$ is shown in Figs. 2(a) and 2(b) for the theoretical and 
experimental cases, respectively. We used $N=30$ iterations and $M=4$ constraint images corresponding to $\alpha=0,22^{\circ}, 44^{\circ}$ and $90^{\circ}$; see left panels of Figs. 2(a) and $2(\mathrm{~b})$. The wavefield $g(\mathbf{r})$ retrieved from the numerically simulated data [right panel of Fig. 2(a)] coincides with the reference beam, $f(\mathbf{r})=\mathrm{LG}_{4,1}(\mathbf{r}, \bar{w})$, as follows from the correlation parameter $\eta=0.999$. In the experiment, the exact generation of $f(\mathbf{r})$ is not possible due to limitations of the holographic encoding and the lack of flatness of the SLM displays that yields to phase aberrations in the retrieved phase of $g(\mathbf{r})$; see Fig. 2(b). Although the experimental reference signal $f(\mathbf{r})$ differs from the ideal one, the measured constraint images are in good agreement with the theoretical ones. As we have mentioned, in the case of unknown $f(\mathbf{r})$, the RMS error of the measured constraint images with respect the estimated ones can be used to estimate the accuracy of the recovery process. Due to the noise in the measurements, the RMS error is about $23 \%$, which is reasonably good.

In the partially coherent case the number of constraint images, in the interval $\alpha \in\left[0,90^{\circ}\right]$, required for successful beam recovery is increased up to $M=12$. In general, the number of the needed constraints increases as the beam's coherence decreases in order to compensate the loss of information in every constraint image caused by partial coherence; see left panel of Fig. 2 . Theoretical and experimental reconstructions of $g(\mathbf{r})$ for the partially coherent case are shown in Figs. 2(c) and 2(d), respectively. The measured constraint images (left panel of Fig. 2; see Media 1) and the retrieved fields (right panel of Fig. 2) are in good agreement with the theoretical ones. In the simulation, the correlation between the retrieved wavefield $g(\mathbf{r})$ [Fig. 2(c)] and the reference one is $\eta=0.98$, while in the experiment the RMS error is 21\%. In contrast, as observed in Fig. 2(e), the reconstruction fails using the conventional retrieval algorithm ignoring that the beam is partially coherent. Indeed, the correlation drastically drops to $\eta=0.41$ in the simulation, while in the experiment the RMS error increased up to $56 \%$. These results illustrate that the beam's coher- ence must be taken into account for correct information recovery [i.e., $f(\mathbf{r})]$ and therefore for quantitative imaging applications.

The proposed iterative algorithm can be adapted to recover not only $f(\mathbf{r})$ but also the DoC of SMBs. This requires an additional iterative routine in the algorithm for the DoC determination, as for example the RichardsonLucy deconvolution (RLD) reported in [13]. In our case, RLD is performed in each iteration step (i) as follows: $\tilde{\gamma}_{\alpha(m)}^{(k+1)}=\tilde{\gamma}_{\alpha(m)}^{(k)} \times\left(P^{(k, m)}\left(-\mathbf{r}_{o}\right) \otimes H^{(k, m)}\left(\mathbf{r}_{o}\right)\right)$, with $k=1, \ldots, K$ being the RLD iteration index and $H^{(k, m)}\left(\mathbf{r}_{o}\right)=I_{\mathrm{pc}}^{\alpha(m)} /\left(P^{(k, m)} \otimes \tilde{\gamma}_{\alpha(m)}^{(k)}\right)$, where $P^{(k, m)}\left(\mathbf{r}_{o}\right)=$ $2 \mathcal{I}_{c,(k)}^{\alpha(m)}-\mathcal{I}_{c,(k-1)}^{\alpha(m)}$ is a combination of the current and previous estimate of the coherent intensity. As an initial estimate, $\tilde{\gamma}_{\alpha(m)}^{(1)}$, an arbitrary Gaussian function was used, which can be applied for any SMB because its DoC is such that $\tilde{\gamma}_{\alpha}$ is a real-positive function. Applying this modified algorithm with $K=3$, the wavefield $g(\mathbf{r})$ [Fig. 3(a)] and the DoC-whose amplitude profile is displayed in Fig. 3(b), red line-are successfully recovered from the experimental data. The retrieved $g(\mathbf{r})$ apparently agrees with the one [Fig. 2(d)] obtained using the known DoC [its profile is also displayed in Fig. 3(b), blue line]; however, their correlation is $\eta=0.85$. The main difference between them is the noise present in the retrieved phases that artificially decreases the correlation. Nevertheless, the DoC retrieved via RLD has a Gaussian-like profile with $w_{c}=0.43 \mathrm{~mm}$, agreeing with the expected value $w_{c}=0.45 \mathrm{~mm}$.

In our case, the developed algorithm permits SMB recovery within 2 min (Intel Core i7, Matlab R2013a). To quantitatively evaluate its performance, let us discuss the evolution of the RMS error of the estimated constraint images with respect the measured ones. The RMS error given as a function of the number of iterations $(n)$ is displayed in Fig. 3(c). Here we consider the reconstruction of the coherent vortex $\mathrm{LG}_{4,1}$ (green color) and the partially coherent one, using both the known DoC (blue color) and the DoC retrieved via
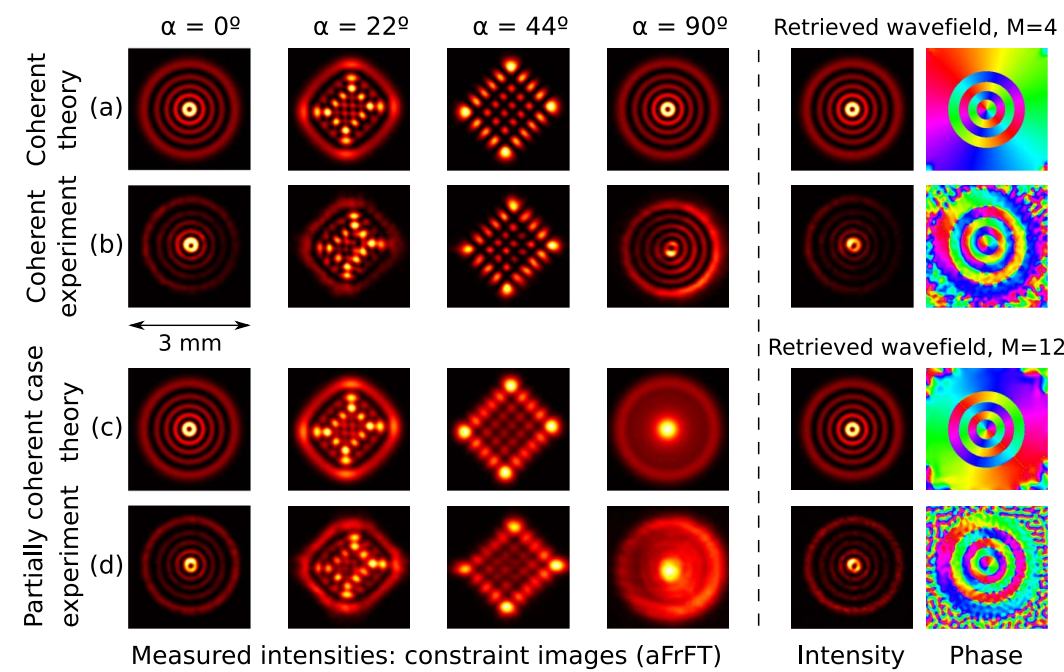

(e)

Retrieved wavefield when ignoring the DoC $(M=12)$

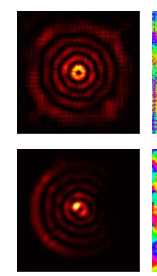

Intensity

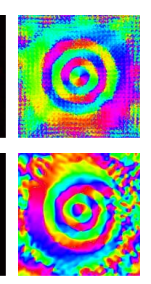

Phase

Fig. 2. Reconstruction of coherent and partially coherent vortices corresponding to LG $_{4,1}$ mode. (Left panel) Constraint images measured for the (a), (b) coherent and (c), (d) partially coherent cases; see Media 1 . The reconstructed beams, using $M=4$ and 12 constraint images, are displayed in the second panel. (e) Beam recovery is significantly degraded when the DoC is ignored. 


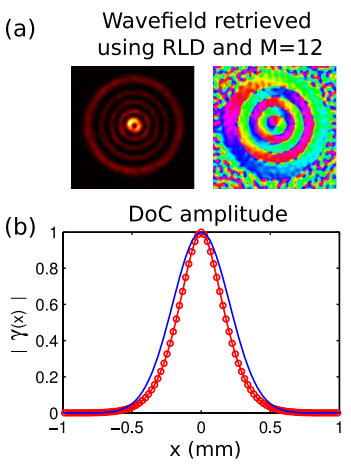

(c)

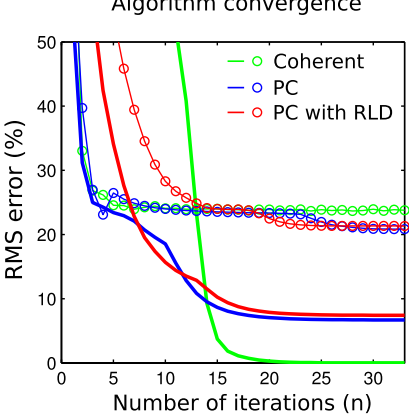

Fig. 3. (a) Vortex $\mathrm{LG}_{4,1}$ experimentally reconstructed using the DoC retrieved via RLD with aFrFT. (b) Amplitude profile of the retrieved DoC (red color) and the expected one (blue color). (c) Convergence of the iterative algorithm for coherent and partially coherent (PC) illumination: simulation (continuous line) and experimental (scatterplot) results.

RLD (red color). The same number of constraints $(M=12)$ in the range $\alpha \in\left[0,90^{\circ}\right]$ was used in each case. In the simulation the convergence is reached at $0 \%$ of RMS error for the coherent case (continuous green line), while for the partially coherent beam in both cases, the known DoC and the one retrieved via RLD, the value of RMS error is $7 \%$. We have found that a lower value of RMS error can be obtained by using more constraint images because more information about the wavefield and DoC is available. In the experiment [see scatterplots in Fig. 3(c)] the RMS error is higher (about 20\% in the coherent and partially coherent cases) due to the noise in the intensity measurements as well as limited sampling (e.g., pixel size and only 256 intensity gray levels).

In summary, the main strategies to retrieve essential information of partially coherent beams have been determined. The developed technique allows for fast and accurate recovery of both the DoC and the beam's spatial structure required, for example, in quantitative phase imaging [e.g., in microscopy, where $f(\mathbf{r})$ plays the role of the object's image] and beam characterization.

The Spanish Ministerio de Economía y Competitividad is acknowledged for funding the project TEC2011-23629.

\section{References}

1. A. C. Schell, "The multiple plate antenna," Ph.D. thesis (Massachusetts Institute of Technology, 1961).

2. J. W. Goodman, Statistical Optics, 1st ed. (WileyInterscience, 2000).

3. F. Wang, Y. Cai, and O. Korotkova, Opt. Express 17, 22366 (2009).

4. T. Alieva, J. A. Rodrigo, A. Cámara, and E. Abramochkin, J. Opt. Soc. Am. A 30, 2237 (2013).

5. G. Gibson, J. Courtial, M. Padgett, M. Vasnetsov, V. Pas'ko, S. Barnett, and S. Franke-Arnold, Opt. Express 12, 5448 (2004).

6. J. C. Ricklin and F. M. Davidson, J. Opt. Soc. Am. A 19, 1794 (2002).

7. J. Li and B. Lü, J. Opt. A 11, 045710 (2009).

8. M. G. Raymer, M. Beck, and D. F. McAlister, Phys. Rev. Lett. 72, 1137 (1994).

9. A. Cámara, J. A. Rodrigo, and T. Alieva, Opt. Express 21, 13169 (2013).

10. J. A. Rodrigo, T. Alieva, and M. L. Calvo, Opt. Express 17, 4976 (2009).

11. J. A. Rodrigo, T. Alieva, G. Cristóbal, and M. L. Calvo, Opt. Express 19, 18621 (2011).

12. C. A. Henderson, G. J. Williams, A. G. Peele, H. M. Quiney, and K. A. Nugent, Opt. Express 17, 11905 (2009).

13. J. Clark, X. Huang, R. Harder, and I. Robinson, Nat. Commun. 3, 993 (2012).

14. J. Arlt, K. Dholakia, L. Allen, and M. J. Padgett, J. Mod. Opt. 45, 1231 (1998). 